
Deficiencies in p70S6K Activation

Glanz, Stephan ; Mirsaidi, Ali ; López-Fagundo, Cristina ; Filliat, Gladys ; Tiaden, André N ; Richards, Peter J

DOI: https://doi.org/10.1089/scd.2015.0368

Posted at the Zurich Open Repository and Archive, University of Zurich

ZORA URL: https://doi.org/10.5167/uzh-127468

Journal Article

Accepted Version

Originally published at:

Glanz, Stephan; Mirsaidi, Ali; López-Fagundo, Cristina; Filliat, Gladys; Tiaden, André N; Richards, Peter J (2016). Loss-of-Function of HtrA1 Abrogates All-TransRetinoic Acid-Induced Osteogenic Differentiation of Mouse Adipose-Derived Stromal Cells Through Deficiencies in p70S6K Activation. Stem Cells and Development, 25(9):687-698.

DOI: https://doi.org/10.1089/scd.2015.0368 


\title{
Loss-of-Function of HtrA1 Abrogates All-Trans Retinoic Acid-Induced Osteogenic Differentiation of Mouse Adipose-Derived Stromal Cells Through Deficiencies in p70S6K Activation
}

Stephan Glanz ${ }^{1,2^{*}}$, Ali Mirsaidi ${ }^{1, *}$, Cristina López-Fagundo ${ }^{1}$, Gladys Filliat ${ }^{1,2}$, André N. Tiaden $^{1}$, Peter J. Richards ${ }^{1,2}$

${ }^{1}$ Bone and Stem Cell Research Group, CABMM, University of Zurich, 8057 Zurich, Switzerland, ${ }^{2}$ Zurich Center for Integrative Human Physiology (ZIHP), University of Zurich, 8057 Zurich, Switzerland. *Authors contributed equally.

\begin{abstract}
Author Email addresses: Stephan.glanz@cabmm.uzh.ch, ali.mirsaidi@cabmm.uzh.ch, clopezfagundo@gmail.com,_gladys.filliat@,cabmm.uzh.ch,_nicki.tiaden@cabmm.uzh.ch, peter.richards@cabmm.uzh.ch
\end{abstract}

Running title: Regulation of mASC Osteogenesis by HtrA1

To whom correspondence should be addressed: Dr. Peter J. Richards, Center for Applied Biotechnology and Molecular Medicine, University of Zürich, Winterthurerstrasse 190, Zürich 8057, Switzerland, Tel:+41-44-635-3800, Email: peter.richards@cabmm.uzh.ch 


\section{Footnotes and Abbreviations}

The abbreviations used are: ALP, alkaline phosphatase; ATRA, all-trans retinoic acid; BMP, bone morphogenetic protein; BMPR, BMP receptor; GAPDH, glyceraldehyde-3-phosphate dehydrogenase; 4E-BP1, eukaryotic initiation factor 4E binding protein 1; hBMSC, human bone marrow stromal cells; HtrA1, high temperature requirement protease A1; mASC, mouse adiposederived stromal cells; mBMSC, mouse bone marrow stromal cells; mTOR, mammalian target of rapamycin; p70S6K, p70 ribosomal protein S6 kinase; rpS6, ribosomal protein S6; SAM, senescence-accelerated mouse; TSC2, Tuberous sclerosis complex 2. 


\begin{abstract}
All-trans retinoic acid (ATRA) is a potent inducer of osteogenic differentiation in mouse adipose-derived stromal cells (mASCs), although the underlying mechanisms responsible for its mode of action have yet to be completely elucidated. High temperature requirement protease A1 (HtrA1) is a newly recognized modulator of human multipotent stromal cell (MSC) osteogenesis and as such, may play a role in regulating ATRA-dependent osteogenic differentiation of mASCs. In the current study, we assessed the influence of small interfering RNA (siRNA)induced repression of HtrA1 production on mASC osteogenesis and examined its effects on ATRA-mediated mammalian target of rapamycin (mTOR) signaling. Inhibition of HtrA1 production in osteogenic mASCs resulted in a significant reduction of alkaline phosphatase (ALP) activity and mineralized matrix formation. Western blot analyses revealed the rapid activation of Akt (Ser473) and p70S6K (Thr389) in ATRA-treated mASCs, and that levels of phosphorylated p70S6K were noticeably reduced in HtrA1-deficient mASCs. Further studies using mTOR inhibitor rapamycin and siRNA specific for the p70S6K gene Rps6kb1 confirmed ATRA-mediated mASC osteogenesis as being dependent on p70S6K activation. Finally, transfection of cells with a constitutively active rapamycin-resistant p70S6K mutant could restore the mineralizing capacity of HtrA1-deficient mASCs. These findings therefore lend further support for HtrA1 as a positive mediator of MSC osteogenesis and provide new insights into the molecular mode of action of ATRA in regulating mASC lineage commitment.
\end{abstract}




\section{Introduction}

Efficient osteogenic induction of mouse adipose-derived stromal cells (mASCs) is reliant on the actions of all-trans retinoic acid (ATRA), the carboxylic acid form of vitamin A [1-7]. This is in contrast to mouse and human bone marrow stromal cells (BMSCs), where dexamethasone is primarily used to instigate osteogenesis through upregulation of four and a half LIM domains 2 (FHL2) and activation of Wnt/ $\beta$ catenin signaling [8]. ATRA's ability to influence osteoblast differentiation has been observed in several different cell systems and is considered to be largely dependent on the concentration of ATRA used. Whilst ATRA acts to enhance osteogenesis at micromolar concentrations $[1-7,9,10]$, at nanomolar concentrations, it has been shown to inhibit both osteoblast gene expression and mineralization [11-13]. The concentration of ATRA used to stimulate mASC osteogenesis in vitro is generally within the range of 1 to $5 \mu \mathrm{M}$, where it acts to enhance the expression of several osteogenic markers including alkaline phosphatase $(A l p l)$ and osteopontin (Spp1), and to induce mineralization of mASC-derived osteoblasts [3, 4]. In addition, ATRA's ability to direct mASCs along the osteoblast lineage in vitro has also been exploited for the purpose of enhancing mASC-induced new bone formation in vivo. Priming of mASCs with ATRA prior to their implantation into mouse calvarial defects resulted in accelerated bone regeneration as compared to mice treated with unstimulated mASCs [14]. However, the mechanisms through which ATRA instigates its osteogenic effects in these cells remain unclear. Findings from studies investigating the combined effects of ATRA and bone morphogenetic protein (BMP)-2 on mASC osteogenesis suggested that ATRA's primary function was to regulate BMP signaling through enhanced BMP receptor (BMPR) expression [1]. However, ATRA also has the ability to induce osteogenic differentiation of mASCs in the 
absence of exogenous BMP-2 [2-7]. Therefore, it's likely that in addition to BMP signaling, ATRA targets other pathways critically involved in regulating mASC osteogenesis.

We have previously identified high temperature requirement protease A1 (HtrA1) as a novel mediator of human BMSC (hBMSC) differentiation, where it acts to enhance osteogenesis and subsequent mineralization by differentiating bone-forming cells [7]. Furthermore, HtrAl expression is upregulated in mASCs in response to ATRA-containing osteogenic induction medium [7]. HtrA1 is a member of the HtrA family of serine proteases and has been linked to various biological processes by virtue of its ability to interact with numerous intracellular and extracellular substrates [15]. Tuberous sclerosis complex 2 (TSC2) was the first cytoplasmic HtrA1 substrate to be identified, and its degradation by HtrA1 was shown to result in activation of the mammalian target of rapamycin (mTOR) pathway as confirmed by alterations in the phosphorylation of downstream targets eukaryotic initiation factor 4E binding protein 1 (4EBP1) and p70 ribosomal protein S6 kinase (p70S6K) [16]. This bears particular significance with regards to mASC osteogenesis, based on the fact that mTOR signaling plays a positive role in the osteogenic induction of several cell types including BMSCs [17-19]. However, no studies have yet sort to investigate its involvement in mediating the osteoinductive effects of ATRA on mASCs, or whether HtrA1's ability to influence mTOR signaling plays a role in determining mASC osteogenic potential. In the present study, we investigated the role of HtrAl in the ATRA-dependent differentiation of mASCs into mineral-forming bone cells and assessed its influence over mTOR signaling events during the course of mASC osteogenesis. 


\section{Materials and Methods}

\section{Materials}

Antibodies specific for non-phosphorylated Akt, mTOR, p70S6K, 4E-BP1 and rpS6; phosphorylated Akt (Ser473), mTOR (Ser2448), p70S6K (Thr389),4E-BP1 (Thr37/46) and rpS6 (Ser235/236) were all purchased from Cell Signaling, BioConcept (Allschwil, Switzerland). Mouse monoclonal anti-tubulin was from Sigma-Aldrich (Buchs, Switzerland). Mouse HAprobe antibody and anti-GAPDH were from Santa Cruz Biotechnology, LabForce AG (Muttenz, Switzerland). A polyclonal anti-HtrA1 antibody was generated as previously described [20]. HRP-labeled secondary antibodies specific for mouse or rabbit IgG were purchased from Jackson ImmunoResearch (Suffolk, UK). Rapamycin was purchased from Enzo Life Science (Lausen, Switzerland). BMP-2 was kindly donated by Prof. Franz Weber (University of Zurich), and ATRA, dexamethasone and PF-4708671 were from Sigma-Aldrich. The expression plasmids pRK7-HA-S6K1-F5A-E389-R3A (Addgene plasmid \# 8991) and pRK7-HA-S6K1-KR (Addgene plasmid \# 8985), were kind gifts from John Blenis [21].

\section{Isolation and Culture of $m A S C s$}

Primary mASCs were isolated from SAM mice as previously described [4, 5]. All animal research procedures were approved by the Animal Experimentation Committee of the Veterinary Office of the Canton of Zurich, Switzerland and followed the guidelines of the Swiss Federal Veterinary Office for the use and care of laboratory animals. Briefly, subcutaneous inguinal fat pads were removed and digested in Hepes buffer containing $0.1 \%$ collagenase A (Roche Diagnostics, Rotkreuz, Switzerland) and $0.2 \%$ bovine serum albumin for $40 \mathrm{~min}$ at $37^{\circ} \mathrm{C}$. Adherent stromal cells were maintained in complete medium consisting of Dulbecco's modified 
eagle medium (DMEM-low glucose, with GlutaMAX) (Life Technologies, Zug, Switzerland), supplemented with 10\% fetal bovine serum (FBS) (Bioswisstec, Schaffhausen, Switzerland) and antibiotics. Supernatant was replaced after 1 day with fresh complete medium and cells were used between passage 1 and 4 following initial analysis for mesenchymal and hematopoietic cell markers by flow cytometry as previously described [4].

\section{Osteogenic Differentiation of $m A S C s$}

mASCs were plated at 5000 cells $/ \mathrm{cm}^{2}$ and incubated in alpha-minimum essential medium $(\alpha-$ MEM) (Life Technologies), supplemented with 10\% FBS (Bioswisstec), $50 \mu \mathrm{M}$ L-ascorbic acid 2-phosphate sesquimagnesium salt hydrate, $10 \mathrm{mM} \beta$-glycerophosphate and either $5 \mu \mathrm{M} \underline{\mathrm{ATRA}}$ or $100 \mathrm{nM}$ dexamethasone and $100 \mathrm{ng} / \mathrm{ml} \mathrm{BMP-2}$ for up to 21 days with regular changes of medium as previously described [4]. Where indicated, PF-4708671 was also added to mASCs undergoing osteogenic differentiation in order to assess the influence of S6K1 inhibition on ATRA-dependent osteogenic induction. Alkaline phosphatase (ALP) activity was quantified in cell lysates using p-nitrophenylphosphate (pNPP) liquid substrate (Sigma-Aldrich) and values normalized to total protein content and reaction time as previously described [4]. Mineralization was visualized using Alizarin red and the amount of staining determined by measuring optical densities at $570 \mathrm{~nm}$ following extraction using 10\% cetylpyridinium chloride (Sigma-Aldrich). Optical densities were then converted to micromoles $(\mu \mathrm{M})$ of Alizarin red using a standard curve and normalized to cell number. The mean cell number was determined by automated counting of 4',6-diamidino-2-phenylindole (DAPI) stained nuclei in at least 6 random fields of view. Images were captured on a Leica DMI 6000 inverted fluorescence microscope (Leica Microsystems, 
Heerbrugg, Switzerland). Image processing and nuclear counts were performed using NIH ImageJ software.

\section{Quantitative Reverse Transcription PCR (RT-qPCR)}

Total RNA was isolated from mASCs and purified using TRIzol reagent (Invitrogen AG, Basel, Switzerland) according to the manufacturer's instructions. RNA $(0.5 \mu \mathrm{g})$ was reverse transcribed to cDNA using Superscript II (Invitrogen AG) and random hexanucleotide primers (Promega AG, Dübendorf, Switzerland). Quantification of mRNA expression was performed with TaqMan Gene Expression Assays (Applied Biosystems, Rotkreuz, Switzerland) specific for HtrA1 (Mm00479887), Rps6kb1 (Mm01310033), Alpl (Mm01187117) and Sppl (Mm01611440) using the StepOnePlus Real-Time PCR System (Applied Biosystems) and values normalized to Mrps12 (Mm00488728) mRNA levels and presented as fold change according to the $2^{-\Delta \Delta C T}$ method. Each $10 \mu 1$ reaction consisted of 1x TaqMan Fast Universal PCR Master Mix (Applied Biosystems), 1x TaqMan Gene Expression Assay and 10 ng cDNA (based upon initial RNA concentrations). All reactions were performed in triplicate in fast optical 96-well reaction plates (Applied Biosystems) at $95^{\circ} \mathrm{C}$ for 20 seconds and 40 cycles of $95^{\circ} \mathrm{C}$ for 1 second and $60^{\circ} \mathrm{C}$ for 20 seconds.

\section{Western Blot Analysis}

Total cellular protein was extracted from mASCs using CelLytic M (Sigma-Aldrich) containing protease and phosphatase inhibitor cocktails (Sigma-Aldrich). For the analysis of HtrA1 in mASC supernatants, cells were treated for 3 days with osteogenic induction medium and then for a further $24 \mathrm{~h}$ in fresh FCS-free osteogenic induction medium before harvesting and 
concentrating supernatants 30-fold using Amicon Ultra-15, $10 \mathrm{kDa}$ mwco filter units (Millipore). In each case, protein amounts were quantified using BioRad Protein Assay (BioRad, Reinach, Switzerland). Protein samples were boiled for $5 \mathrm{~min}$ in loading buffer (50 mM Tris-HCl, $\mathrm{pH} 6.8$, $2 \%$ SDS, $10 \%$ glycerol, $100 \mathrm{mM}$ DTT, $0.002 \%$ Bromophenol blue) and equal amounts of protein loaded onto $12 \%$ SDS-PAGE gels. Protein was then electroblotted onto PVDF membranes using the Trans-Blot Turbo blotting system (BioRad) and incubated in 5\% skimmed milk, $50 \mathrm{mM}$ Tris$\mathrm{HCl}, \mathrm{pH} 7.6,150 \mathrm{mM} \mathrm{NaCl}, 0.1 \%$ Tween 20 (TBST) for $1 \mathrm{~h}$ at room temperature. Membranes were then incubated overnight at $4^{\circ} \mathrm{C}$ with primary antibodies specific for $\mathrm{HtrAl}$ or

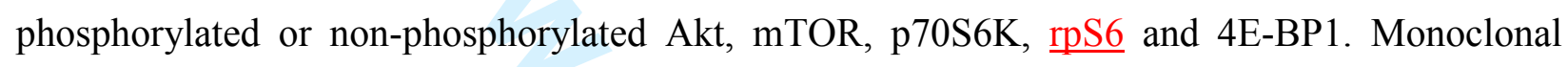
mouse anti-tubulin or anti-GAPDH were used to control for equal protein loading of cell lysates. Coomassie blue staining was used to control for equal protein loading of cell supernatants. After washing in TBST three times for $5 \mathrm{~min}$ each, membranes were incubated with a HRP-conjugated anti-mouse or anti-rabbit $\operatorname{IgG}\left(1: 10^{\prime} 000\right)$ for $1 \mathrm{~h}$ at room temperature. Following a further washing step, peroxidase activity was detected using SuperSignal West Pico Chemiluminescent Substrate (Thermo Scientific, Lausanne, Switzerland). Protein levels were quantified using NIH ImageJ software. Phosphorylated and non-phosphorylated protein values were first normalized to tubulin loading control and then the phosphorylation to total protein ratio calculated using the normalized values.

\section{Small Interfering RNA (siRNA) Studies}

Specific knock down of gene expression was performed with Silencer Select siRNA (Ambion, Life Technologies) specific for HtrA1 (s80180) or Rps6kb1 (s91055) using previously described methods [7]. Briefly, mASCs (1x10 cells) were transfected with $20 \mathrm{nM}$ of targeted siRNA or 
negative control siRNA (Negative Control-1) using the NEON Transfection System (Life Technologies). Following transfection, cells were seeded in cell culture plates with fresh growth medium (without antibiotics) and incubated for $24 \mathrm{~h}$ at $37^{\circ} \mathrm{C}, 5 \% \mathrm{CO}_{2}$. Medium was then replaced with either fresh growth medium or osteogenic differentiation medium and total RNA or protein harvested at selected time points for further analysis. The effects of siRNA mediated gene knockdown on osteogenic-induced mASC ALP activity and mineralization was determined using the ALP activity assay and Alizarin Red staining respectively.

siRNA and Plasmid Co-Transfection

mASCs were transfected with Silencer Select siRNA specific for HtrA1 or Negative Control-1 and $1 \mu \mathrm{g}$ of mammalian expression plasmid pRK7-HA-S6K1-F5A-E389-R3A (constitutively active p70S6K), pRK7-HA-S6K1-KR (kinase inactive p70S6K) or empty control plasmid pcDNA3 using the NEON Transfection System as described above. After $24 \mathrm{~h}$, cells were induced to undergo osteogenesis and mineralization quantified after 21 days using Alizarin red staining as described above.

\section{Statistical Analysis

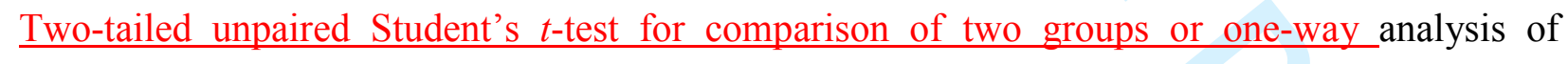 variance (ANOVA) with Tukey's post-hoc test for multiple group comparisons were performed using SPSS19.0 (SPSS Inc., Chicago, IL). In all cases, a $p$-value of $<0.05$ was considered statistically significant.}




\section{Results}

We have previously demonstrated that HtrA1 plays a vital role in the regulation of osteogenesis in hBMSCs [7]. In the current report, we further investigated this property of HtrA1 in ATRAstimulated mASCs and aimed to establish its role in regulating mASC osteogenesis and mASCderived osteoblast mineralization.

\section{HtrA1 Deficiency Impairs ATRA-Mediated mASC Osteogenic Differentiation}

In order to examine the influence of loss-of-function of HtrAl on the osteogenic capacity of mASCs in response to treatment with osteogenic medium containing ATRA, we analyzed mineral production by mASC-derived osteoblasts, as well as ALP expression and enzyme activity. Analysis of HtrA1 in mASC supernatants by Western blot confirmed HtrA1 protein production to be effectively reduced in osteogenic mASCs treated with siRNAs specific for HtrAl (Fig. 1A). We next assessed the influence of loss-of-function of HtrA1 on the osteogenic potential of mASCs. mASCs treated with control siRNA underwent efficient osteoblastogenesis and mineralization following stimulation with osteogenic medium for 10 days, as determined by Alizarin red staining (Fig. 1B). However, Alizarin red staining of osteogenic-induced mASCs in which HtrA1 had previously been depleted was noticeably reduced (Fig. 1B). Similarly, HtrA1 deficiency also resulted in significant reductions in Alizarin red staining in mASCs induced to undergo osteogenic differentiation in response to BMP-2 treatment (supplementary Fig. S1). Further quantitative analysis of extracted Alizarin red stain revealed the mineralizing capabilities of HtrA1-deficient mASC-derived osteoblasts to be significantly impaired $(p<0.001)$ as compared to siControl (Fig. 1C). In accordance with our previous findings [7], ATRA-mediated osteogenic induction of mASCs resulted in significant increases in both HtrAl (Fig. 1D) and Alpl 
(Fig. 1E) expression levels in a time dependent manner. Similarly, ALP enzyme activity was also significantly enhanced in response to osteogenic induction at all time points tested (Fig. 1F). As expected, HtrAl knockdown of mASCs significantly suppressed HtrAl expression in osteogenic mASCs over the course of the study (Fig. 1D). ATRA-mediated increases in Alpl expression levels were also significantly impaired in HtrA1-deficient mASCs (Fig. 1E) and were accompanied by significant decreases in ALP enzymatic activity at all time points tested (Fig. 1F). These findings therefore demonstrate a functional role for HtrA1 in regulating ATRAmediated mASC osteogenesis and in the generation of a mineralized matrix by mASC-derived osteoblasts.

\section{HtrA1 Deficiency Impairs ATRA-Mediated p70S6K Activation in mASCs}

Having demonstrated HtrA1 to be a necessary component for efficient mASC osteogenesis, we next considered its potential mode of action. Based on HtrA1's previously reported role in the activation of mTOR targets p70S6K and 4E-BP1 in tumor cell lines [16], we assessed the possible influence of HtrA1 deficiency on mTOR signaling in ATRA-stimulated mASCs. As no studies have yet sought to determine the effects of ATRA-mediated osteogenic induction on mTOR signaling in mASCs, we initially performed a series of Western blot analyses to ascertain the activation status of kinases located both upstream and downstream of mTOR. Phosphorylation levels of Akt (Ser473), mTOR (Ser2448), p70S6K (Thr389) and 4E-BP1 (Thr37/46) were assessed in mASCs over the course of $2 \mathrm{~h}$ following ATRA-mediated osteogenic induction. A noticeable increase in Akt and p70S6K phosphorylation was already evident in osteogenic-induced mASCs after only $10 \mathrm{~min}$ and had reduced to basal levels by $1 \mathrm{~h}$ (Fig. 2A). However, basal levels of phosphorylated mTOR were only minimally affected after 10 
min, and 4E-BP1 remained relatively unchanged at all time points tested. We next proceeded to investigate the influence of HtrAl silencing on Akt/mTOR/p70S6K/4E-BP1 phosphorylation in differentiating mASCs. Western blot analysis of cell lysates from HtrA1-deficient mASCs revealed no reduction in phospho-Akt levels, and only minor reductions in phospho-mTOR and 4EBP1 levels as compared to siControl (Fig. 2B). By comparison however, HtrA1-deficient mASCs demonstrated marked reductions in the levels of phosphorylated p70S6K and rpS6. These results are therefore suggestive of ATRA-mediated p70S6K activation as being a potential HtrA1 target and a means by which it could influence ATRA-dependent mASC osteogenesis.

\section{Loss-of-Function of p 70S6K Impairs mASC Osteogenesis}

In order to address the functional relevance of p70S6K activation in the context of mASC osteogenesis, we next assessed the effects of p70S6K inhibition on ALP expression and matrix mineralization in mASCs undergoing ATRA-mediated osteogenic differentiation. Short-term treatment of mASCs with the mTOR inhibitor rapamycin prior to osteogenic induction markedly reduced mTOR phosphorylation and completely abolished p70S6K phosphorylation, whilst Akt phosphorylation levels remained unaffected (Fig. 3A). Next, we evaluated the effects of longterm exposure of mASCs to rapamycin with regards to their ability to differentiate into mineralizing osteoblasts. Alizarin red staining of osteogenic mASCs was significantly reduced by rapamycin treatment in a concentration dependent manner (Fig. 3B and C), thus confirming that mTOR signaling was required for mASC-derived osteoblastogenesis.

Further investigations employing siRNA mediated knockdown of the p70S6K gene Rps6kb1 were also performed in order to assess the effects of specifically inhibiting p70S6K activity on mASC osteogenesis. p70S6K protein and activity levels were noticeably reduced in 
Rps6kb1-deficient mASCs after short-term osteogenic induction (Fig. 4A). We next investigated the effects of Rps $6 \mathrm{kbl}$ knockdown on mASC-derived osteoblast mineralization using Alizarin red staining. A marked reduction in Alizarin red staining was observed in Rps6kbl-deficient mASCs after 14 days of culture in osteogenic medium (Fig. 4B). Further quantitative analysis of extracted Alizarin red stain revealed the mineralizing capabilities of p70S6K-deficient mASCderived osteoblasts to be significantly impaired as compared to siControl $(p<0.001)$ (Fig. 4C). In support of these findings, significant reductions in Alizarin red staining of mASC-derived osteoblasts were also observed in cultures treated with the specific S6K1 inhibitor PF-4708671 (supplementary Fig. S2). Gene expression analyses of mASCs undergoing osteogenesis confirmed efficient Rps6kbl knockdown throughout the course of the study and revealed a significant reduction in $R p s 6 \mathrm{kbl}$ gene expression at day 14 in siControl in response to osteogenic induction (Fig. 4D). Furthermore, the expression levels of osteogenic markers Alpl (Fig. 4E) and osteopontin (Spp1) (Fig. 4F) in mASCs undergoing osteogenesis were significantly reduced in p70S6K-deficient cells. These results clearly identify p70S6K activation as being a necessary requirement for efficient osteogenic differentiation of mASCs and provide a potential means through which HtrA1 may regulate ATRA-mediated mASC osteogenesis.

Restoration of HtrA1-Deficient mASC Osteogenesis Using a Constitutively Active p70S6K Mutant

In view of the fact that mASC osteogenesis is dependent on both HtrA1 and p70S6K, and that loss-of-function of HtrA1 impairs p70S6K activation, we sought to determine whether transfection of mASCs with a constitutively active p70S6K mutant could relieve the detrimental effects of HtrA1 deficiencies on mASC osteogenesis. mASCs were transfected with either an 
empty plasmid, or plasmids encoding a rapamycin-resistant constitutively active (p70S6KCA) or kinase inactive (p70S6KKI) HA-tagged p70S6K [21]. The results shown in Fig. 5A are representative of the controls used to confirm that the plasmids encoding p70S6KCA and p70S6KKI were performing as expected. Both protein products were labeled with an HA-tag, and so plasmid-mediated protein expression could be accurately and specifically detected using an anti-HA antibody. As shown in the top lane of Fig. 5A, HA-labeled protein is evident in cells treated with plasmids encoding p70S6KCA and p70S6KKI as expected, but no signal is detected in cells treated with empty plasmid as no HA-labeled protein has been produced. Cells transfected with empty plasmid therefore serve as an additional control to confirm Western blot specificity and the robustness of the cell transfection system used. The p70S6K substrate $\mathrm{rpS6}$, is phosphorylated by activated p70S6K and therefore represents a useful means by which to visualize p70S6K activation by Western blot. As such, inhibition of p70S6K activity by rapamycin treatment is expected to result in reduced phosphorylated $\mathrm{rpS6}$ (p-rpS6) levels. Indeed, upon rapamycin treatment of mASCs transfected with empty plasmid or plasmid encoding p70S6KKI, we observed a noticeable reduction in p-rpS6 levels in cells. This would be expected as neither the empty plasmid nor the kinase inactive p70S6KKI can generate active, rapamycin resistant $\mathrm{p} 70 \mathrm{~S} 6 \mathrm{~K}$. However, cells expressing the rapamycin resistant, active P70S6KCA protein can still phosphorylate rpS6 even in the presence of rapamycin. Therefore, these results are confirmation that mASCs, when transfected with plasmid DNA, can produce the relevant $\mathrm{p} 70 \mathrm{~S} 6 \mathrm{~K}$ proteins and that they are either active (p70S6KCA) or inactive (p70S6KKI). RT-qPCR confirmed that siRNA mediated reduction of HtrAl mRNA expression was unaffected in cells co-transfected with plasmid DNA (Fig. 5B). Next, we transfected HtrA1-deficient mASCs with empty plasmid, p70S6KCA or p70S6KKI and assessed their ability to influence 
mASC-derived osteoblast formation through quantification of Alizarin red staining after 21 days. The assumption was that reduced p70S6K activity in HtrA1-deficient mASCs could be compensated for through expression of the constitutively active p70S6K (p70S6KCA), and thereby restore osteogenic potential. Indeed, as shown in Fig. 5C and 5D, Alizarin red staining was restored in HtrA1-deficient mASCs transfected with p70S6KCA. The kinase inactive (KI) p70S6K (p70S6KKI) was included to control as accurately as possible for the introduction of plasmid DNA into the cells and expression of p70S6K protein. It differs with respect to p70S6KCA in that it is not active, and so should not compensate for the reductions in p70S6K activity in HtrA1-deficient mASCs. This was indeed the case, as shown in Fig. 5C and 5D where Alizarin red staining could not be restored in HtrA1-deficient mASCs transfected with p70S6KKI. Empty plasmid was also included as an additional control, and demonstrated no influence over mASC osteogenesis as expected. Taken together, these findings identify p70S6K as being of critical importance in ATRA-mediated mASC osteogenesis and that activation of p70S6K is reliant, at least in part, on the actions of HtrA1.

\section{Discussion}

mASCs represent a readily available source of osteoprogenitor cells, which unlike mBMSCs, have the advantage of being able to sustain a high level of osteogenic differentiation potential with age and under conditions of low bone quality [4, 22-25]. Subsequently, mASCs are fast becoming the preferred choice for stem cell-based approaches in bone tissue engineering [26, 27]. Certainly, results from our previous studies have confirmed that mASCs harvested from SAMP6 mice, a model for senile osteoporosis, have the capability of increasing bone quality when re-injected back into SAMP6 tibia [5]. However, despite their widespread usage, the 
underlying mechanisms through which mASC osteogenic differentiation is controlled remains incompletely understood. In the current report, we identify the serine protease HtrAl as being a positive regulator of ATRA-induced mASC osteogenesis and mASC-derived osteoblast mineralization. Furthermore, we provide evidence, which supports $\mathrm{p} 70 \mathrm{~S} 6 \mathrm{~K}$ as playing a role in mediating the pro-osteogenic effects of HtrA1 in mASCs in response to ATRA.

Mammalian HtrA1 was originally identified by Zumbrunn and Trueb [28] and has since been implicated in numerous biological processes and diseases [15, 29]. Findings from our own studies have revealed HtrA1 to be a potent modulator of hBMSC multipotency as evidenced by its ability to inhibit adipogenesis and stimulate osteogenesis [7]. However, several studies also exist in which HtrA1 has been classified as a negative regulator of osteogenesis both in vitro and in vivo $[30,31]$. Clearly therefore, further investigations are required in order to clarify HtrA1's function in osteogenesis and to ascertain its potential mechanism of action. Here we provide further evidence in support of HtrA1's role as a positive regulator of MSC osteogenesis.

HtrA1 is classified as a secreted serine protease and as such, its influence over cellular processes is largely thought to be due to its extracellular actions $[7,20,32]$. However, it is also equally likely that HtrA1 instigates many of its effects intracellularly. Indeed, HtrA1 has been shown to interact with and functionally regulate several intracellular substrates including tubulin [33], proTGF $\beta 1$ [34], tau [35], X-linked inhibitor of apoptosis protein (XIAP) [36] and TSC2 [16]. In the context of the present study, HtrA1's regulatory influence over TSC2 activity holds particular relevance given the importance of mTOR signaling in stem cell multipotency [17-19].

The mTOR protein makes up the catalytic subunit of two separate complexes, namely mTOR complex 1 (mTORC1) and mTOR complex 2 (mTORC2) [37]. mTORC1 functions to control cell growth and protein synthesis through phosphorylation of 4E-BP1 and p70S6K, and 
has been implicated in osteoblast differentiation [17, 38-40]. mTORC1 is negatively regulated by the GTPase-activating protein TSC1/2 complex and as such, relies on the actions of Akt for its activation through phosphorylation of TSC2 [41]. Although several studies have demonstrated activation of the Akt/mTOR signaling pathway in response to ATRA [42-44], no investigations have yet been undertaken to examine Akt/mTOR activation in ATRA-stimulated mASCs or to evaluate its consequences for their commitment towards osteoblasts. Our findings have demonstrated that the $\mathrm{Akt} / \mathrm{mTOR} / \mathrm{p} 70 \mathrm{~S} 6 \mathrm{~K}$ pathway is rapidly activated in mASCs in response to ATRA. Similar rapid increases in several other kinase cascades have previously been demonstrated in various cell systems in response to ATRA [45-48]. Such effects are considered to be independent of the classical genomic effects of ATRA, and are instead regulated through atypical, non-genomic events possibly through interactions with membrane-associated retinoic acid receptors (RARs) [49]. The ability of the mTOR inhibitor rapamycin to completely suppress ATRA-mediated p70S6K activation in these cells suggests that despite the minimal increases in phospho-mTOR (Ser2448), ATRA-mediated p70S6K activation in mASCs is rapamycin sensitive and as such, reliant on mTOR signaling. It is important to note that although p70S6K is well recognized as a downstream target of mTOR, phosphorylation of mTOR at Ser2448 is also considered to represent a feedback signal from p70S6K [50]. If indeed the case, then ATRAmediated p70S6K activation in mASCs may possibly be reliant on increases in mTOR activity through the phosphorylation of sites other than Ser2448. Certainly, mTOR has been reported to have several potential phosphorylation sites whose functions remain largely undefined [51]. As with rapamycin treatment, loss-of-function of HtrAl also resulted in reductions in mTOR and p70S6K phosphorylation in the absence of any changes in phospho-Akt. However, in contrast to 
rapamycin, the removal of HtrA1 was unable to completely abolish mTOR or p70S6K phosphorylation.

Although p70S6K activity is considered to be of paramount importance in determining mASC adipocyte lineage commitment [52], its functional role in mASC osteogenesis has not yet been established. Our findings from studies using the mTOR inhibitor rapamycin, along with siRNA-dependent inhibition of Rps $6 \mathrm{kbl}$ gene expression, confirmed that the mTOR/p70S6K signaling pathway was indeed an essential requirement for efficient mASC osteogenesis and mASC-derived osteoblast mineralization. As far as we are aware, this is the first report to demonstrate such a role for mTOR/p70S6K in ATRA-mediated mASC osteogenesis. Therefore, these studies identified both HtrA1 and mTOR/p70S6K as being important regulators of ATRAmediated mASC osteogenesis. However, it was still unclear as to whether HtrA1's ability to regulate p70S6K phosphorylation in response to ATRA was directly related to its pro-osteogenic effects. We therefore performed a study in which we introduced plasmids encoding DNA for either constitutively active or kinase inactive mutants of p70S6K into HtrA1-deficient mASCs in an attempt to rescue osteoblastogenesis. Indeed, our results revealed that the mineralizing capacity of HtrA1-deficient mASC-derived osteoblasts could be fully restored when cells were engineered to express the constitutively active p70S6K mutant.

These findings therefore identify p70S6K activation as a regulatory target of HtrA1 and an important event in mediating HtrAl's pro-osteogenic effects in ATRA-stimulated mASCs. However, based on the fact that mTOR phosphorylation at Ser2448 may be the result of feedback regulation by $\mathrm{p} 70 \mathrm{~S} 6 \mathrm{~K}$, further investigations are needed in order to determine whether

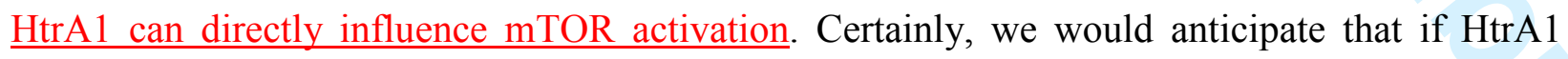
were acting to regulate p70S6K activity through its interaction with TSC2 [16], then reductions 
in mTOR activity would be apparent [53]. However, the suggestion that the TSC-complex may in fact regulate p70S6K independently of mTOR [54], may offer an additional means by which HtrA1 could activate p70S6K without the need for alterations in mTOR activity. Alternatively, HtrA1 may act to regulate p70S6K phosphorylation through mTOR, but in a TSC2-independent manner. Certainly, mTOR is not solely reliant on TSC2 inhibition for its activation as confirmed by studies in which Akt was shown to activate mTOR by relieving the inhibitory effects of proline-rich Akt/PKB substrate $40 \mathrm{kDa}$ (PRAS40) on mTORC1 [55], and more recently, through its ability to promote mTORC1 phosphorylation at Ser1415 via the actions of IkB kinase alpha $(\mathrm{IKK} \alpha)[56]$. Further studies are therefore required in order to ascertain the involvement of TSC2 and mTOR in mediating the effects of HtrA1 on p70S6K phosphorylation in osteogenic mASCs.

Despite us having now demonstrated HtrA1 to be a positive regulator of both mASC and $\underline{\text { hBMSC }[7] \text { osteogenesis, its role in mediating bone formation remains highly controversial. This }}$ is highlighted by findings from previous studies in which HtrA1 was deemed to be a negative $\underline{\text { regulator of osteogenesis in stromal cells derived from long-term bone marrow cultures }}$ (LTBMCs) [31] and mouse 2T3 osteoblasts [57]. By contrast however, a more recent report has identified HtrA1 as being a necessary requirement for the osteogenic differentiation of periodontal ligament cells [58]. One possible explanation for such discrepancies may lie in the fact that in each of these studies, a different cell culture system was used. If indeed the case, this would imply that HtrA1 acts to mediate cell-specific responses to osteogenic stimuli, the result of which may impart an inhibitory or enhancing effect on osteogenic induction. Interestingly, our new findings also demonstrate that HtrA1's involvement in regulating mASC osteogenesis extends beyond it's ability to influence ATRA-mediated osteogenic induction as evidenced by $\underline{\text { significant reductions in mineral formation in HtrA1-deficient mASC cultures stimulated with }}$ 
BMP-2. In order to try and further elucidate HtrAl's role in regulating bone formation, investigations have also been conducted in mice with a targeted gene deletion of HtrAl [30]. However, although HtrA1-deficient mice displayed significant increases in several bone parameters, the influence of such changes on bone quality and strength remain to be determined. Furthermore, no studies were undertaken to address the possible involvement of compensatory mechanisms in HtrA1 deficient mice, such as the upregulation of other members of the HtrA family (e.g. HtrA3 and HtrA4), which may also have an influence on bone formation. Clearly, more in-depth investigations are required in order to reconcile these conflicting studies and thereby help clarify HtrA1's role as a modulator of bone formation.

In summary, we have identified p70S6K as an important regulator of mASC osteogenesis, being activated in response to ATRA via pathways involving mTOR and HtrAl (Fig. 6). As such, it is proposed that HtrA1 represents a newly identified positive regulator of ATRA-mediated mASC osteogenesis and mASC-derived osteoblast mineralization.

\section{Acknowledgements}

SG and GF were supported by SNSF grants 31003A_134935 and 31003A_156313. AM and ANT were supported by the Uniscientia Foundation and Forschungskredit University of Zurich. CLF was supported by the Whitaker International Program.

\section{Disclosure Statement}

The authors declare that they have no competing financial interests.

\section{References}


1. Wan DC, YY Shi, RP Nacamuli, N Quarto, KM Lyons, and MT Longaker. (2006). Osteogenic differentiation of mouse adipose-derived adult stromal cells requires retinoic acid and bone morphogenetic protein receptor type IB signaling. Proc Natl Acad Sci U S A $103: 12335-12340$.

2. Wan DC, MT Siedhoff, MD Kwan, RP Nacamuli, BM Wu, and MT Longaker. (2007). Refining retinoic acid stimulation for osteogenic differentiation of murine adipose-derived adult stromal cells. Tissue Eng 13:1623-631.

3. Malladi P, Y Xu, GP Yang, and MT Longaker. (2006). Functions of vitamin D, retinoic acid, and dexamethasone in mouse adipose-derived mesenchymal cells. Tissue Eng 12:20312040.

4. Mirsaidi A, KN Kleinhans, M Rimann, AN Tiaden, M Stauber, KL Rudolph, and PJ Richards. (2012). Telomere length, telomerase activity and osteogenic differentiation are maintained in adipose-derived stromal cells from senile osteoporotic SAMP6 mice. J Tissue Eng Regen Med 6:378-390.

5. Mirsaidi A, K Genelin, JR Vetsch, S Stanger, F Theiss, RA Lindtner, B von Rechenberg, M Blauth, R Müller, GA Kuhn, S Hofmann Boss, HL Ebner, and PJ Richards. (2014). Therapeutic potential of adipose-derived stromal cells in age-related osteoporosis. Biomaterials 35:7326-7335.

6. Mirsaidi A, AN Tiaden, and PJ Richards. (2013). Preparation and osteogenic differentiation of scaffold-free mouse adipose-derived stromal cell microtissue spheroids (ASC-MT). Curr Protoc Stem Cell Biol 27: Unit 2B.5.

7. Tiaden AN, M Breiden, A Mirsaidi, FA Weber, G Bahrenberg, S Glanz, P Cinelli, M Ehrmann, and PJ Richards. (2012). Human serine protease HTRA1 positively regulates 
osteogenesis of human bone marrow-derived mesenchymal stem cells and mineralization of differentiating bone-forming cells through the modulation of extracellular matrix protein. Stem Cells 30:2271-2282.

8. Hamidouche Z, E Haÿ, P Vaudin, P Charbord, R Schüle, PJ Marie, and O Fromigué. (2008). FHL2 mediates dexamethasone-induced mesenchymal cell differentiation into osteoblasts by activating Wnt/beta-catenin signaling-dependent Runx2 expression. FASEB J 22:38133822.

9. Hisada K, K Hata, F Ichida, T Matsubara, H Orimo, T Nakano, H Yatani, R Nishimura, and T Yoneda. (2013). Retinoic acid regulates commitment of undifferentiated mesenchymal stem cells into osteoblasts and adipocytes. J Bone Miner Metab 31:53-63.

10. Song HM, RP Nacamuli, W Xia, AS Bari, YY Shi, TD Fang, and MT Longaker. (2005). High-dose retinoic acid modulates rat calvarial osteoblast biology. J Cell Physiol 202:255262.

11. Ohishi K, S Nishikawa, T Nagata, N Yamauchi, H Shinohara, J Kido, and H Ishida. (1995). Physiological concentrations of retinoic acid suppress the osteoblastic differentiation of fetal rat calvaria cells in vitro. Eur J Endocrinol 133:335-341.

12. Iba K, H Chiba, T Yamashita, S Ishii, and N Sawada. (2001). Phase-independent inhibition by retinoic acid of mineralization correlated with loss of tetranectin expression in a human osteoblastic cell line. Cell Struct Funct 26:227-233.

13. Lind T, A Sundqvist, L Hu, G Pejler, G Andersson, A Jacobson, and H Melhus. (2013). Vitamin a is a negative regulator of osteoblast mineralization. PLoS One 8:e82388.

14. Cowan CM, OO Aalami, YY Shi, YF Chou, C Mari, R Thomas, N Quarto, RP Nacamuli, CH Contag, B Wu, and MT Longaker. (2005). Bone morphogenetic protein 2 and retinoic 
acid accelerate in vivo bone formation, osteoclast recruitment, and bone turnover. Tissue Eng 11:645-58.

15. Clausen T, M Kaiser, R Huber, and M Ehrmann. (2011). HTRA proteases: regulated proteolysis in protein quality control. Nat Rev Mol Cell Biol 12:152-162.

16. Campioni M, A Severino, L Manente, IL Tuduce, S Toldo, M Caraglia, S Crispi, M Ehrmann, X He, J Maguire, M De Falco, A De Luca, V Shridhar, and A Baldi. (2010). The serine protease HtrA1 specifically interacts and degrades the tuberous sclerosis complex 2 protein. Mol Cancer Res 8:1248-1260.

17. Singha UK, Y Jiang, S Yu, M Luo, Y Lu, J Zhang, and G Xiao. (2008). Rapamycin inhibits osteoblast proliferation and differentiation in MC3T3-E1 cells and primary mouse bone marrow stromal cells. J Cell Biochem 103:434-446.

18. Isomoto S, K Hattori, H Ohgushi, H Nakajima, Y Tanaka, and Y Takakura. (2007). Rapamycin as an inhibitor of osteogenic differentiation in bone marrow-derived mesenchymal stem cells. J Orthop Sci 12:83-88.

19. Shoba LN, and JC Lee. (2003). Inhibition of phosphatidylinositol 3-kinase and p70S6 kinase blocks osteogenic protein-1 induction of alkaline phosphatase activity in fetal rat calvaria cells. J Cell Biochem 88:1247-1255.

20. Grau S, PJ Richards, B Kerr, C Hughes, B Caterson, AS Williams, U Junker, SA Jones, T Clausen, and M Ehrmann. (2006). The role of human HtrA1 in arthritic disease J Biol Chem 281:6124-6129.

21. Schalm SS, and J Blenis. (2002). Identification of a conserved motif required for mTOR signaling. Curr Biol 12:632-639. 
22. Schipper BM, KG Marra, W Zhang, AD Donnenberg, and JP Rubin. (2008). Regional anatomic and age effects on cell function of human adipose-derived stem cells. Ann Plast Surg 60:538-544.

23. Zhu M, E Kohan, J Bradley, M Hedrick, P Benhaim, and P Zuk. (2009). The effect of age on osteogenic, adipogenic and proliferative potential of female adipose-derived stem cells. J Tissue Eng Regen Med 3:290-301.

24. Khan WS, AB Adesida, SR Tew, JG Andrew, and TE Hardingham. (2009). The epitope characterization and the osteogenic differentiation potential of human fat pad-derived stem cells is maintained with ageing in later life. Injury 40:150-157.

25. Shi YY, RP Nacamuli, A Salim, and MT Longaker. (2005). The osteogenic potential of adipose-derived mesenchymal cells is maintained with aging. Plast Reconstr Surg 116:16861696.

26. Liu HY, JF Chiou, AT Wu, CY Tsai, JD Leu, LL Ting, MF Wang, HY Chen, CT Lin, DF Williams, and WP Deng. (2012). The effect of diminished osteogenic signals on reduced osteoporosis recovery in aged mice and the potential therapeutic use of adipose-derived stem cells. Biomaterials 33:6105-6112.

27. You L, L Pan, L Chen, JY Chen, X Zhang, Z Lv, and D Fu. (2012). Suppression of zinc finger protein 467 alleviates osteoporosis through promoting differentiation of adipose derived stem cells to osteoblasts. J Trans1 Med 10:11.

28. Zumbrunn J, and B Trueb. (1996). Primary structure of a putative serine protease specific for IGF-binding proteins. FEBS Lett 398:187-92.

29. Tiaden AN, and PJ Richards. (2013). The emerging roles of HTRA1 in musculoskeletal disease. Am J Pathol 182:1482-488. 
30. Graham JR, A Chamberland, Q Lin, XJ Li, D Dai, W Zeng, MS Ryan, MA RiveraBermúdez, CR Flannery, and Z Yang. (2013). Serine protease HTRA1 antagonizes transforming growth factor- $\beta$ signaling by cleaving its receptors and loss of HTRA1 in vivo enhances bone formation. PLoS One. 8:e74094.

31. Wu X, SM Chim, V Kuek, BS Lim, ST Chow, J Zhao, S Yang, V Rosen, J Tickner, and J Xu. (2014). HtrA1 is upregulated during RANKL-induced osteoclastogenesis, and negatively regulates osteoblast differentiation and BMP2-induced Smad1/5/8, ERK and p38 phosphorylation. FEBS Lett 588:143-50.

32. Tiaden AN, M Klawitter, V Lux, A Mirsaidi, G Bahrenberg, S Glanz, L Quero, T Liebscher, K Wuertz, M Ehrmann, and PJ Richards. (2012). Detrimental role for human high temperature requirement serine protease A1 (HTRA1) in the pathogenesis of intervertebral disc (IVD) degeneration. J Biol Chem 287:21335-21345.

33. Chien J, T Ota, G Aletti, R Shridhar, M Boccellino, L Quagliuolo, A Baldi, and V Shridhar. (2009). Serine protease HtrA1 associates with microtubules and inhibits cell migration. Mol Cell Biol 29:4177-4187.

34. Shiga A, H Nozaki, A Yokoseki, M Nihonmatsu, H Kawata, T Kato, A Koyama, K Arima, M Ikeda, S Katada, Y Toyoshima, H Takahashi, A Tanaka, I Nakano, T Ikeuchi, M Nishizawa, and O Onodera. (2011). Cerebral small-vessel disease protein HTRA1 controls the amount of TGF- $\beta 1$ via cleavage of proTGF- $\beta 1$. Hum Mol Genet 20:1800-1810.

35. Tennstaedt A, S Pöpsel, L Truebestein, P Hauske, A Brockmann, N Schmidt, I Irle, B Sacca, CM Niemeyer, R Brandt, H Ksiezak-Reding, AL Tirniceriu, R Egensperger, A Baldi, L Dehmelt, M Kaiser, R Huber, T Clausen, and M Ehrmann. (2012). Human high temperature 
requirement serine protease A1 (HTRA1) degrades tau protein aggregates. J Biol Chem 287:20931-20941.

36. He X, A Khurana, JL Maguire, J Chien, and V Shridhar. (2012). HtrA1 sensitizes ovarian cancer cells to cisplatin-induced cytotoxicity by targeting XIAP for degradation. Int J Cancer 130:1029-1035.

37. Zoncu R, A Efeyan, and DM Sabatini. (2011). mTOR: from growth signal integration to cancer, diabetes and ageing. Nat Rev Mol Cell Biol 12:21-35.

38. Kim J, Y Jung, H Sun, J Joseph, A Mishra, Y Shiozawa, J Wang, PH Krebsbach, and RS Taichman. (2012). Erythropoietin mediated bone formation is regulated by mTOR signaling. J Cell Biochem 113:220-228.

39. Kim JK, J Baker, JE Nor, and EE Hill. (2011). mTor Plays an Important Role in Odontoblast Differentiation. J Endod 37:1081-1085.

40. Tang C-H, DY Lu, TW Tan, WM Fu, and RS Yang. (2007). Ultrasound Induces Hypoxiainducible Factor-1 Activation and Inducible Nitricoxide Synthase Expression through the Integrin/Integrin-linked Kinase/Akt/Mammalian Target of Rapamycin Pathway in Osteoblasts. J Biol Chem 282:25406-25415.

41. Tee AR, BD Manning, PP Roux, LC Cantley, and J Blenis. (2003). Tuberous sclerosis complex gene products, Tuberin and Hamartin, control mTOR signaling by acting as a GTPase-activating protein complex toward Rheb. Curr Biol 13:1259-1268.

42. La L, Y Li, J Smith, A Sassano, S Uddin, S Parmar, MS Tallman, S Minucci, N Hay, and LC Platanias. (2005). Activation of the p70 S6 kinase by all-trans-retinoic acid in acute promyelocytic leukemia cells. Blood 105:1669-1677. 
43. Busada JT, VA Chappell, BA Niedenberger, EP Kaye, BD Keiper, CA Hogarth, and CB Geyer. (2015). Retinoic acid regulates Kit translation during spermatogonial differentiation in the mouse. Dev Biol 397:140-149.

44. Chen N, and JL Napoli. (2008). All-trans-retinoic acid stimulates translation and induces spine formation in hippocampal neurons through a membrane-associated RARalpha. FASEB J 22:236-245.

45. Masiá S, S Alvarez, AR de Lera, and D Barettino. (2007). Rapid, nongenomic actions of retinoic acid on phosphatidylinositol-3-kinase signaling pathway mediated by the retinoic acid receptor. Mol Endocrinol 21:2391-2402.

46. Giannì M, A Bauer, E Garattini, P Chambon, and C Rochette-Egly. (2002). Phosphorylation by p38MAPK and recruitment of SUG-1 are required for RA-induced RAR gamma degradation and transactivation. EMBO J 21:3760-3769.

47. Alsayed Y, S Uddin, N Mahmud, F Lekmine, DV Kalvakolanu, S Minucci, G Bokoch, and LC Platanias. (2001). Activation of Rac1 and the p38 mitogen-activated protein kinase pathway in response to all-trans-retinoic acid. J Biol Chem 276:4012-4019.

48. Bost F, L Caron, I Marchetti, C Dani, Y Le Marchand-Brustel, and B Binétruy. (2002). Retinoic acid activation of the ERK pathway is required for embryonic stem cell commitment into the adipocyte lineage. Biochem J 361:621-627.

49. AI Tanoury Z, A Piskunov, and C Rochette-Egly. (2013). Vitamin A and retinoid signaling: genomic and nongenomic effects. J Lipid Res 54:1761-1775.

50. Chiang GG, and RT Abraham. (2005). Phosphorylation of mammalian target of rapamycin (mTOR) at Ser-2448 is mediated by p70S6 kinase. J Biol Chem 280:25485-25490. 
51. Ekim B, B Magnuson, HA Acosta-Jaquez, JA Keller, EP Feener, and DC Fingar. (2011). mTOR kinase domain phosphorylation promotes mTORC1 signaling, cell growth, and cell cycle progression. Mol Cell Biol 31:2787-27801.

52. Carnevalli LS, K Masuda, F Frigerio, O Le Bacquer, SH Um, V Gandin, I Topisirovic, N Sonenberg, G Thomas, and SC Kozma. (2010). S6K1 plays a critical role in early adipocyte differentiation. Dev Cell 18:763-774.

53. Inoki K, Y Li, T Xu, and KL Guan. (2003). Rheb GTPase is a direct target of TSC2 GAP activity and regulates mTOR signaling. Genes Dev 17:1829-1834.

54. Jaeschke A, J Hartkamp, M Saitoh, W Roworth, T Nobukuni, A Hodges, J Sampson, G Thomas, and R Lamb. (2002). Tuberous sclerosis complex tumor suppressor-mediated S6 kinase inhibition by phosphatidylinositide-3-OH kinase is mTOR independent. J Cell Biol $159: 217-224$.

55. Vander Haar E, SI Lee, S Bandhakavi, TJ Griffin, and DH Kim. (2007). Insulin signalling to mTOR mediated by the Akt/PKB substrate PRAS40. Nat Cell Biol 9:316-323.

56. Dan HC, A Ebbs, M Pasparakis, T Van Dyke, DS Basseres, and AS Baldwin. (2014). Aktdependent activation of mTORC1 complex involves phosphorylation of mTOR (mammalian target of rapamycin) by IкB kinase $\alpha$ (IKK $\alpha)$. J Biol Chem 289:25227-25240.

57. Hadfield KD, CF Rock, CA Inkson, SL Dallas, L Sudre, GA Wallis, RP Boot-Handford, and AE Canfield. (2008). HtrA1 inhibits mineral deposition by osteoblasts: requirement for the protease and PDZ domains. J Biol Chem 283:5928-38.

58. Li R, and Q Zhang. (2015). HtrA1 may regulate the osteogenic differentiation of human periodontal ligament cells by TGF- $\beta 1$. J Mol Histol 46:137-44. 


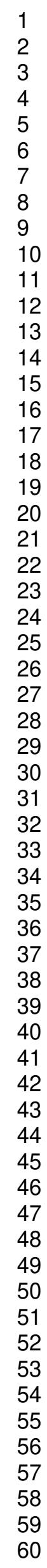


FIG. 1. Effect of HtrAl knockdown on osteogenic induction of mASCs. mASCs were pretreated with control siRNA (siControl) or 2 selected siRNAs targeting HtrAl (siHtrA1-A and siHtrA1-B) for $24 \mathrm{~h}$ and induced to undergo osteogenesis for up to 10 days. (A) Western blot analysis of HtrA1 protein in FCS-free concentrated supernatants from siRNA-treated mASCs after 4 days of osteogenic induction using an antibody specific for HtrA1. Gels were stained with Coomassie Blue to confirm equal protein loading. (B) Alizarin red staining of siRNA-treated mASCs 10 days post osteogenic induction. (C) Extracted Alizarin red stain was quantified and normalized to cell number. ${ }^{*} p<0.001$ as compared to siControl using one-way ANOVA. Gene expression levels of $\mathrm{HtrAl}$ (D) and Alpl (E) were determined in osteogenic mASCs at selected time points using RT-qPCR and the fold change as compared to uninduced mASCs (day 0) determined using the $2^{-\Delta \Delta C T}$ method. (F) ALP enzymatic activity levels were measured in siRNA-treated mASCs over the course of 10 days of osteogenic differentiation using a colorimetric-based ALP activity assay. All values are expressed as mean \pm S.D (triplicates). ${ }^{*} p$ $<0.05,{ }^{* *} p<0.001$ as compared to uninduced mASCs at day $0 ;{ }^{\#} p<0.05,{ }^{\#} p<0.001$ comparison between siControl and siHtrA1 using one-way ANOVA.

FIG. 2. Western blot analysis of kinase activity in mASCs in response to ATRA-mediated osteogenic induction. (A) mASCs were stimulated with osteogenic medium (OM) for up to $2 \mathrm{~h}$ and cell lysates analysed by immunoblotting at selected time points using antibodies specific for phosphorylated or non-phosphorylated Akt, mTOR, p70S6K and 4E-BP1. A mouse anti-tubulin antibody was used to confirm equal protein loading. The influence of osteogenic induction on mASC protein phosphorylation was compared to undifferentiated mASCs (time point 0 min). (B) mASCs were pre-treated with control siRNA (siControl) or siRNA targeting HtrAl (siHtrA1) for 
$24 \mathrm{~h}$ and stimulated with osteogenic medium $(\mathrm{OM})$ for $10 \mathrm{~min}$. Untreated mASCs incubated in growth medium (GM) alone served as non-differentiated controls. Cell lysates were extracted and analysed by immunoblotting using antibodies specific for phosphorylated or nonphosphorylated Akt, mTOR, p70S6K, ribosomal protein $\underline{\text { rpS6 }}$ or 4E-BP1. A mouse anti-tubulin antibody was used to confirm equal protein loading. Fold changes in phosphorylated protein

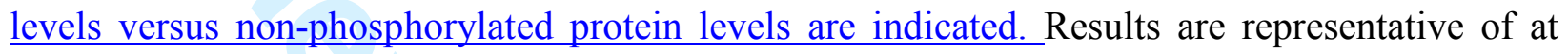
least two separate experiments.

FIG. 3. Effect of rapamycin on osteogenic induction of mASCs. (A) mASCs were incubated with growth medium (GM) or osteogenic medium (OM) for $10 \mathrm{~min}$ and cell lysates analysed by immunoblotting using antibodies specific for phosphorylated or non-phosphorylated Akt, mTOR, or p70S6K. In order to assess the effects of mTOR inhibition on protein activation, cells were also pre-treated for $2 \mathrm{~h}$ with $20 \mathrm{nM}$ rapamycin (Rap) or vehicle $(0.1 \% \mathrm{DMSO})$ prior to osteogenic induction. A mouse anti-tubulin antibody was used to confirm equal protein loading. Results are representative of at least two separate experiments. Fold changes in phosphorylated protein levels versus non-phosphorylated protein levels are indicated. (B) The influence of different concentrations of rapamycin on mASC-derived osteoblast mineralization was determined by Alizarin red staining at day 14. (C) Extracted Alizarin red stain was quantified and normalized to cell number and comparisons made between vehicle-treated and rapamycintreated osteogenic mASCs. All values are expressed as mean \pm S.D (triplicates). ${ }^{*} p<0.001$ as compared to mASCs treated without rapamycin using one-way ANOVA. 
FIG. 4. Effect of Rps6kb1 knockdown on osteogenic induction of mASCs. mASCs were pretreated with control siRNA (siControl) or 2 selected siRNAs targeting Rps6kb1 (siRps6kb1-A and siRps6kb1-B) for $24 \mathrm{~h}$ and induced to undergo osteogenesis for up to 14 days. (A) Western blot analysis of p70S6K and phospho-S6 ribosomal proteinrpS6 (p-rpS6) in siRNA-treated mASCs using antibodies specific for p70S6K and p-rpS6 respectively. A mouse anti-tubulin antibody was used to confirm equal protein loading. (B) Alizarin red staining of siRNA-treated mASCs 14 days post osteogenic induction. (C) Extracted Alizarin red stain was quantified and normalized to cell number. ${ }^{*} p<0.001$ as compared to siControl using one-way ANOVA. (D-F) Gene expression levels of Rps6kb1 (D), Alpl (E) and Sppl (F) were determined in osteogenic mASCs at selected time points using RT-qPCR and the fold change as compared to uninduced mASCs determined using the $2^{-\Delta \Delta C T}$ method. All values are expressed as mean \pm S.D (triplicates). ${ }^{*} p<0.05,{ }^{* *} p<0.01,{ }^{* * *} p<0.001$ as compared to uninduced mASCs at day $0{ }^{*}$ $p<0.01,{ }^{\#} p<0.001$ comparison between siControl and siRps6kb1 using one-way ANOVA.

FIG. 5. Influence of p70S6K activity on siHtrA1-mediated suppression of mASC osteogenesis. (A) mASCs were transfected with empty plasmid, or plasmids encoding influenza hemagglutinin (HA)-tagged constitutively active (p70S6KCA) or kinase inactive (p70S6KKI) p70S6K mutants and immunoblotting performed after $24 \mathrm{~h}$ using an HA-probe antibody (HA-p70S6K) or antiphospho-rpS6 ribesemal protein-antibody (p-rpS6). A mouse anti-GAPDH antibody was used to confirm equal protein loading. (B) The effect of plasmid DNA transfection on siRNA-mediated HtrAl gene silencing in mASCs was determined after $24 \mathrm{~h}$ using RT-qPCR. (C) The influence of plasmid DNA transfection on mASC-derived osteoblast formation in in-HtrA1-deficient mASCs was determined by Alizarin red staining at day 21 post osteogenic induction. (D) Extracted 
Alizarin red stain was quantified and normalized to cell number. All values are expressed as mean \pm S.D (triplicates). ${ }^{*} p<0.01, * * p<0.001$ as compared to siControl/ empty plasmid treated cells; ; ${ }^{\#} p<0.01,{ }^{\#} p<0.001$ comparisons between siHtrA1/p70S6KCA and siHtrA1/p70S6KKI or siHtrA1/p70S6KCA and siHtrA1/empty plasmid using one-way ANOVA.

FIG. 6. Model of ATRA-induced mASC osteogenesis. Based on our findings, we propose that ATRA drives osteoblast commitment of mASCs through activation of p70S6K in a noncanonical and non-genomic manner. This appears not only to be dependent on rapamycinsensitive signaling pathways, but also on the actions of HtrA1. However, further investigations are required in order to determine HtrAl's mechanism of action and to clarify the potential involvement of the HtrA1 substrate TSC2 in mediating its effects on p70S6K activity and mASC osteogenesis.

Supplementary Fig. S1. Effect of HtrAl knockdown on BMP-2-dependent osteogenic induction of mASCs. mASCs were pre-treated with control siRNA (siControl) or an siRNA targeting HtrA1 (siHtrA1) for $24 \mathrm{~h}$ and induced to undergo osteogenesis for 21 days in the absence (untreated) or presence of BMP-2 $(100 \mathrm{ng} / \mathrm{ml})$. (A) Alizarin red staining of siRNA-treated mASCs 21 days post osteogenic induction. (B) Extracted Alizarin red stain was quantified and normalized to cell number. $* p<0.01$ as compared to siControl using Student's $t$-test.

Supplementary Fig. S2. Effect of S6K1 inhibitor PF-4708671 on ATRA-induced osteogenic induction of mASCs. mASCs were induced to undergo osteogenic induction in the absence or presence of PF-4708671 at 10 or $20 \mu \mathrm{M}$ for 15 days. (A) Alizarin red staining of PF-4708671- 
treated mASCs 15 days post osteogenic induction. (B) Extracted Alizarin red stain was quantified and normalized to cell number. ${ }^{*} p<0.01$ as compared to untreated mASCs using one-way ANOVA. 
A

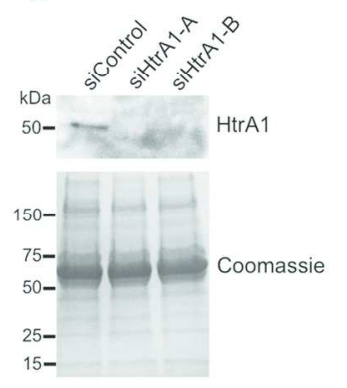

D

$$
\text { - siControl }
$$

口siHtrA1

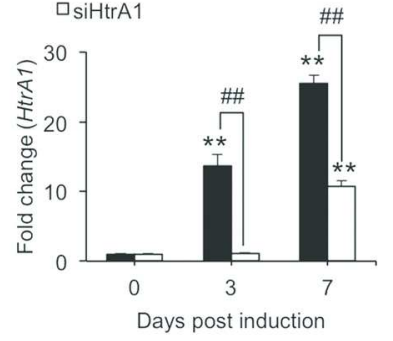

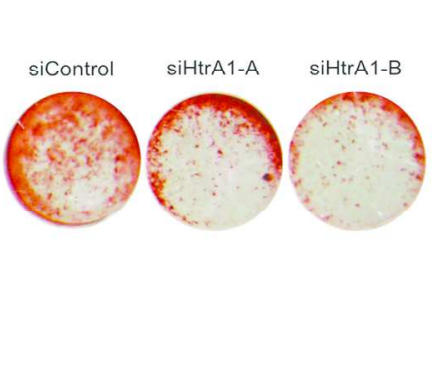

$E$

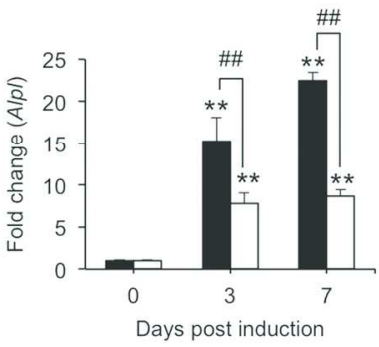

c

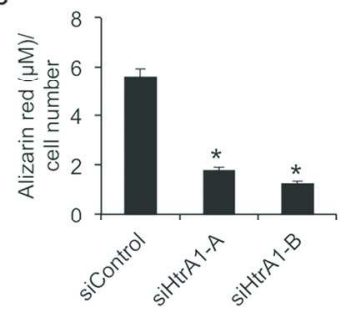

F

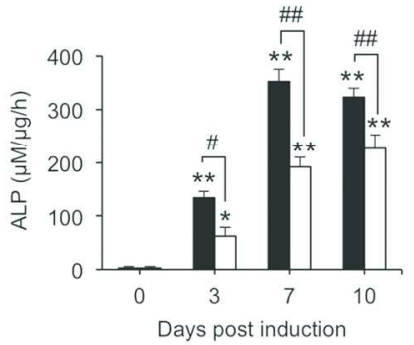

FIG. 1. Effect of HtrA1 knockdown on osteogenic induction of mASCs. mASCs were pre-treated with control siRNA (siControl) or 2 selected siRNAs targeting HtrA1 (siHtrA1-A and siHtrA1-B) for $24 \mathrm{~h}$ and induced to undergo osteogenesis for up to 10 days. (A) Western blot analysis of HtrA1 protein in FCS-free concentrated supernatants from siRNA-treated mASCs after 4 days of osteogenic induction using an antibody specific for HtrA1. Gels were stained with Coomassie Blue to confirm equal protein loading. (B) Alizarin red staining of siRNA-treated mASCs 10 days post osteogenic induction. (C) Extracted Alizarin red stain was quantified and normalized to cell number. $* p<0.001$ as compared to siControl using one-way ANOVA. Gene expression levels of HtrA1 (D) and Alpl (E) were determined in osteogenic mASCs at selected time points using RTqPCR and the fold change as compared to uninduced mASCs (day 0) determined using the $2^{-\triangle \Delta C T}$ method.

(F) ALP enzymatic activity levels were measured in siRNA-treated mASCs over the course of 10 days of osteogenic differentiation using a colorimetric-based ALP activity assay. All values are expressed as mean \pm S.D (triplicates). ${ }^{*} p<0.05, * * p<0.001$ as compared to uninduced mASCs at day 0 ; ${ }^{\#} p<0.05$, \#\# $p<$ 0.001 comparison between siControl and siHtrA1 using one-way ANOVA. $74 \times 56 \mathrm{~mm}(600 \times 600 \mathrm{DPI})$ 


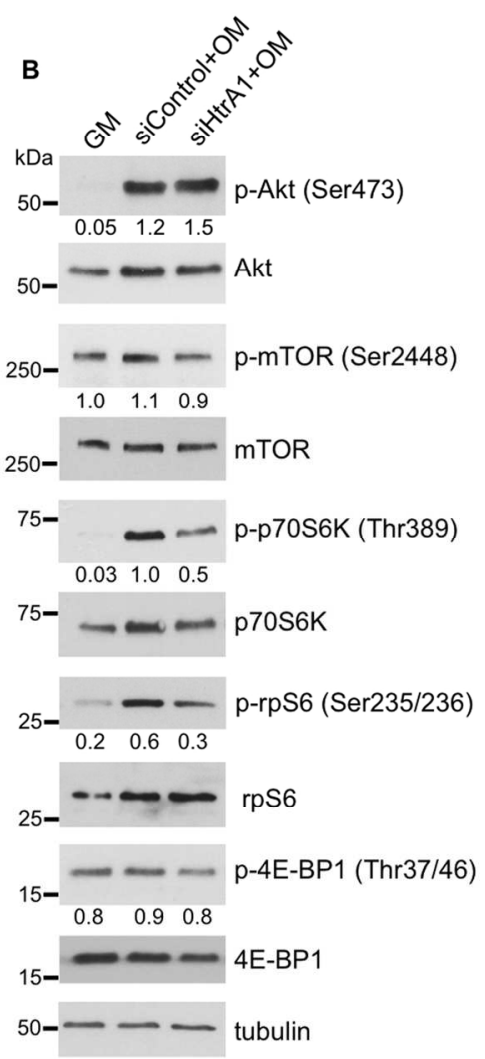

FIG. 2. Western blot analysis of kinase activity in mASCs in response to ATRA-mediated osteogenic induction. (A) mASCs were stimulated with osteogenic medium (OM) for up to $2 \mathrm{~h}$ and cell lysates analysed by immunoblotting at selected time points using antibodies specific for phosphorylated or nonphosphorylated Akt, mTOR, p70S6K and 4E-BP1. A mouse anti-tubulin antibody was used to confirm equal protein loading. The influence of osteogenic induction on mASC protein phosphorylation was compared to undifferentiated mASCs (time point $0 \mathrm{~min}$ ). (B) mASCs were pre-treated with control siRNA (siControl) or siRNA targeting HtrA1 (siHtrA1) for $24 \mathrm{~h}$ and stimulated with osteogenic medium (OM) for 10 min. Untreated mASCs incubated in growth medium (GM) alone served as non-differentiated controls. Cell lysates were extracted and analysed by immunoblotting using antibodies specific for phosphorylated or nonphosphorylated Akt, mTOR, p70S6K, rpS6 or 4E-BP1. A mouse anti-tubulin antibody was used to confirm equal protein loading. Fold changes in phosphorylated protein levels versus non-phosphorylated protein levels are indicated. Results are representative of at least two separate experiments. $82 \times 67 \mathrm{~mm}(600 \times 600 \mathrm{DPI})$ 


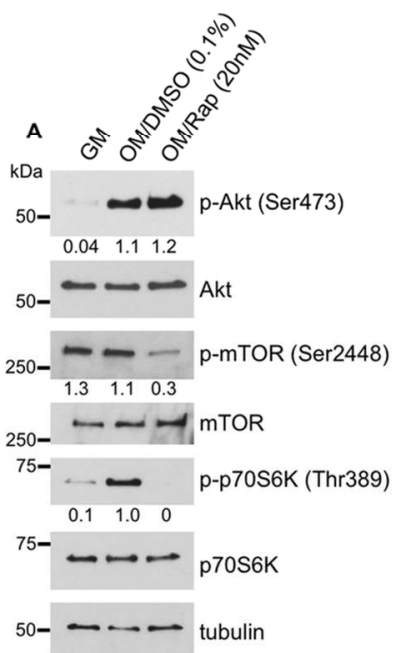

B

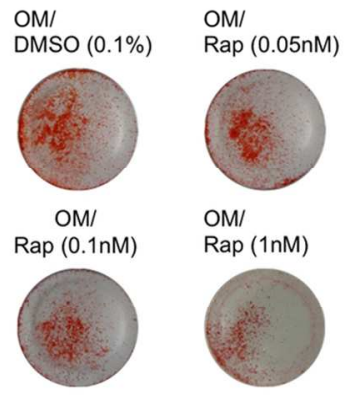

C

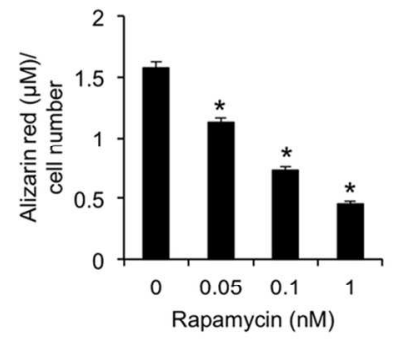

FIG. 3. Effect of rapamycin on osteogenic induction of mASCs. (A) mASCs were incubated with growth medium (GM) or osteogenic medium (OM) for $10 \mathrm{~min}$ and cell lysates analysed by immunoblotting using antibodies specific for phosphorylated or non-phosphorylated Akt, mTOR, or p70S6K. In order to assess the effects of mTOR inhibition on protein activation, cells were also pre-treated for $2 \mathrm{~h}$ with $20 \mathrm{nM}$ rapamycin (Rap) or vehicle $(0.1 \%$ DMSO) prior to osteogenic induction. A mouse anti-tubulin antibody was used to confirm equal protein loading. Results are representative of at least two separate experiments. Fold changes in phosphorylated protein levels versus non-phosphorylated protein levels are indicated. (B) The influence of different concentrations of rapamycin on MASC-derived osteoblast mineralization was determined by Alizarin red staining at day 14. (C) Extracted Alizarin red stain was quantified and normalized to cell number and comparisons made between vehicle-treated and rapamycin-treated osteogenic mASCs. All values are expressed as mean \pm S.D (triplicates). $* p<0.001$ as compared to mASCs treated without rapamycin using one-way ANOVA.

$53 \times 28 \mathrm{~mm}(600 \times 600 \mathrm{DPI})$ 

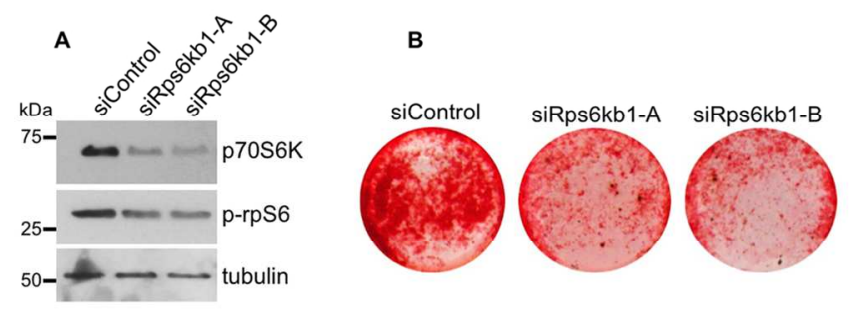

DiControl

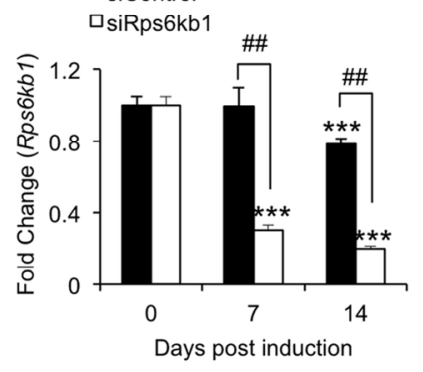

$E$

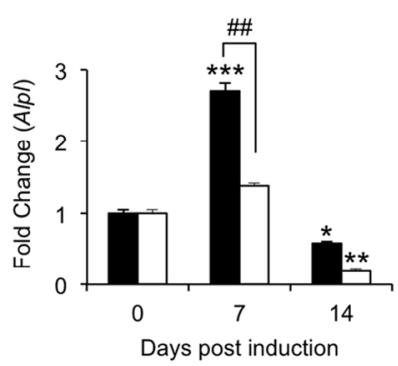

C

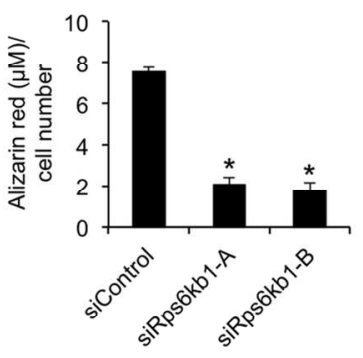

$\mathrm{F}$

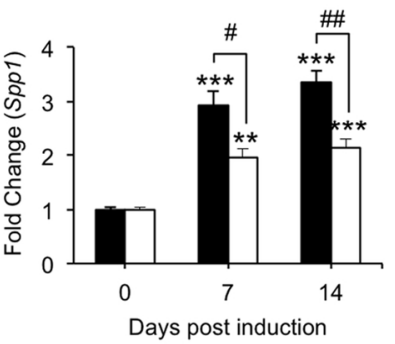

FIG. 4. Effect of Rps6kb1 knockdown on osteogenic induction of mASCs. mASCs were pre-treated with control siRNA (siControl) or 2 selected siRNAs targeting Rps6kb1 (siRps6kb1-A and siRps6kb1-B) for $24 \mathrm{~h}$ and induced to undergo osteogenesis for up to 14 days. (A) Western blot analysis of p70S6K and phosphorpS6 ( $p$-rpS6) in siRNA-treated mASCs using antibodies specific for p70S6K and p-rpS6 respectively. A mouse anti-tubulin antibody was used to confirm equal protein loading. (B) Alizarin red staining of siRNAtreated mASCs 14 days post osteogenic induction. (C) Extracted Alizarin red stain was quantified and normalized to cell number. $* p<0.001$ as compared to siControl using one-way ANOVA. (D-F) Gene expression levels of $R p s 6 k b 1$ (D), $A / p /$ (E) and Spp1 (F) were determined in osteogenic mASCs at selected time points using RT-qPCR and the fold change as compared to uninduced mASCs determined using the $2^{-}$ $\triangle \Delta C T$ method. All values are expressed as mean \pm S.D (triplicates). $* p<0.05, * * p<0.01, * * * p<0.001$ as compared to uninduced mASCs at day $0 ;{ }^{\#} p<0.01$, \#\# $p<0.001$ comparison between siControl and siRps6kb1 using one-way ANOVA.

$67 \times 45 \mathrm{~mm}(600 \times 600$ DPI $)$ 
A

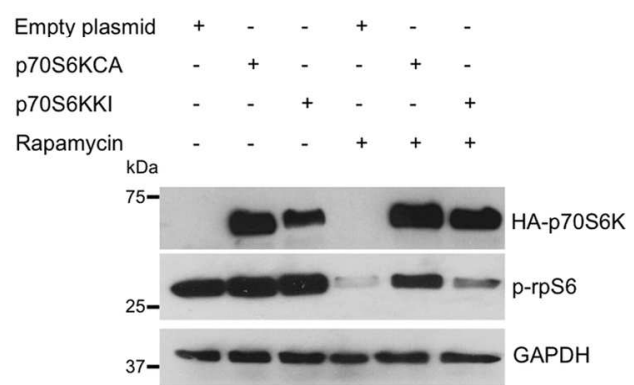

B

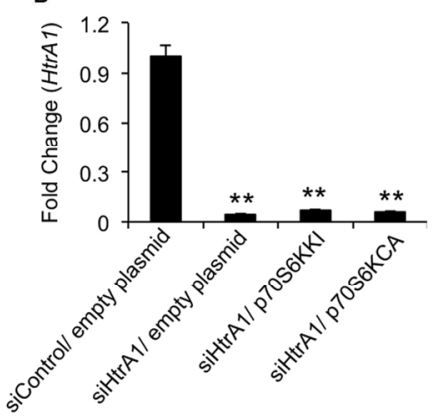

c

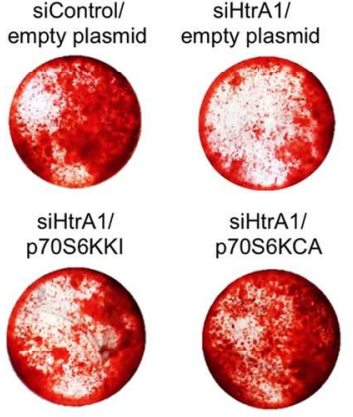

D

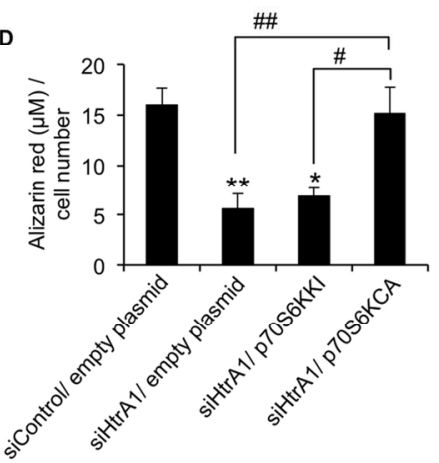

FIG. 5. Influence of $p 70 S 6 \mathrm{~K}$ activity on siHtrA1-mediated suppression of mASC osteogenesis. (A) mASCs were transfected with empty plasmid, or plasmids encoding influenza hemagglutinin (HA)-tagged constitutively active (p70S6KCA) or kinase inactive (p70S6KKI) p70S6K mutants and immunoblotting performed after $24 \mathrm{~h}$ using an HA-probe antibody (HA-p70S6K) or anti-phospho-rpS6 antibody (p-rpS6). A mouse anti-GAPDH antibody was used to confirm equal protein loading. (B) The effect of plasmid DNA transfection on siRNA-mediated HtrA1 gene silencing in mASCs was determined after $24 \mathrm{~h}$ using RT-qPCR.

(C) The influence of plasmid DNA transfection on MASC-derived osteoblast formation in HtrA1-deficient mASCs was determined by Alizarin red staining at day 21 post osteogenic induction. (D) Extracted Alizarin red stain was quantified and normalized to cell number. All values are expressed as mean \pm S.D

(triplicates). * $p<0.01, * * p<0.001$ as compared to siControl/ empty plasmid treated cells; ; ${ }^{*} p<0.01$, \#\# $p<0.001$ comparisons between siHtrA1/p70S6KCA and siHtrA1/p70S6KKI or siHtrA1/p70S6KCA and siHtrA1/empty plasmid using one-way ANOVA. $74 \times 56 \mathrm{~mm}(600 \times 600 \mathrm{DPI})$ 
FIG. 6. Model of ATRA-induced mASC osteogenesis. Based on our findings, we propose that ATRA drives osteoblast commitment of mASCs through activation of p70S6K in a non-canonical and non-genomic manner. This appears not only to be dependent on rapamycin-sensitive signaling pathways, but also on the actions of HtrA1. However, further investigations are required in order to determine HtrA1's mechanism of action and to clarify the potential involvement of the HtrA1 substrate TSC2 in mediating its effects on p70S6K activity and mASC osteogenesis. $119 \times 143 \mathrm{~mm}(600 \times 600 \mathrm{DPI})$ 
A

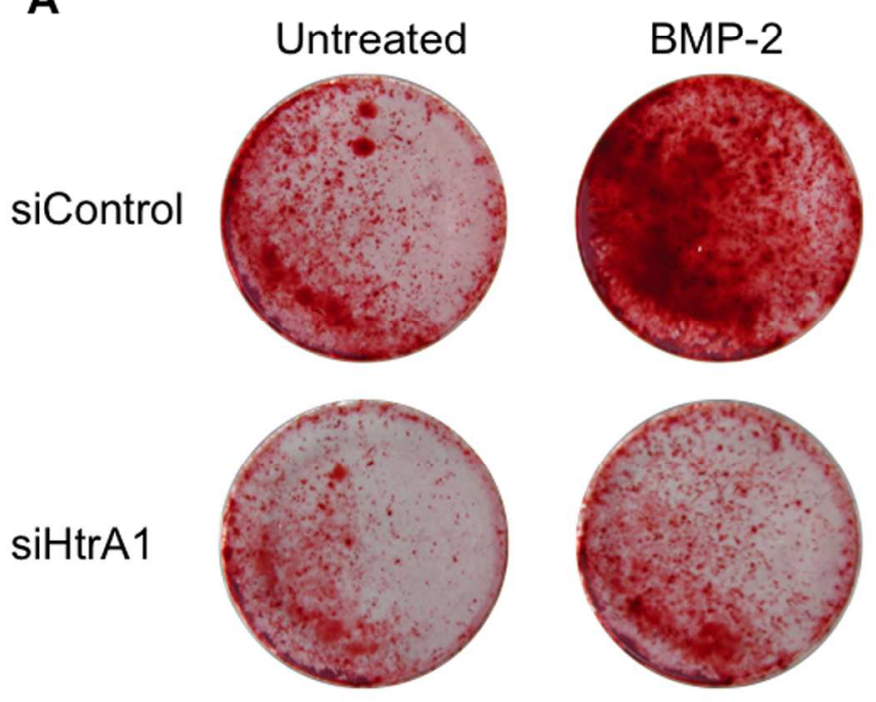

B

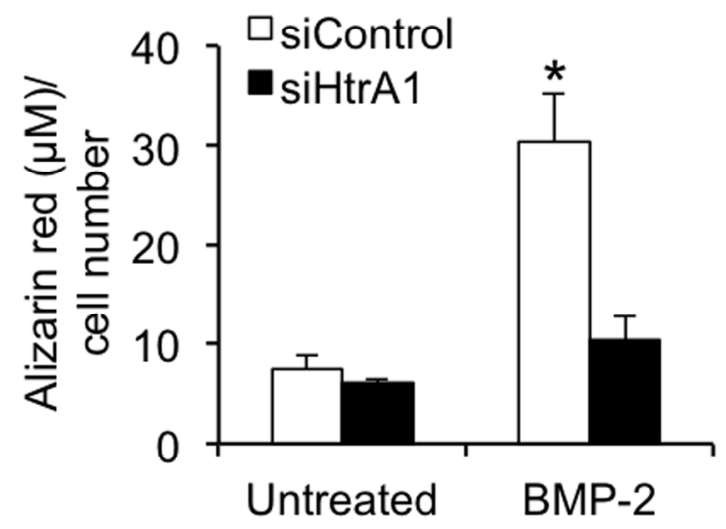

Supplementary FIG. S1. Effect of HtrA1 knockdown on BMP-2-dependent osteogenic induction of mASCs. mASCs were pre-treated with control siRNA (siControl) or an siRNA targeting HtrA1 (siHtrA1) for $24 \mathrm{~h}$ and induced to undergo osteogenesis for 21 days in the absence (untreated) or presence of BMP-2 $(100 \mathrm{ng} / \mathrm{ml}$ ). (A) Alizarin red staining of siRNA-treated mASCs 21 days post osteogenic induction. (B) Extracted Alizarin red stain was quantified and normalized to cell number. ${ }^{*} p<0.01$ as compared to siControl using Student's $t$-test.

$133 \times 177 \mathrm{~mm}(600 \times 600 \mathrm{DPI})$ 
A

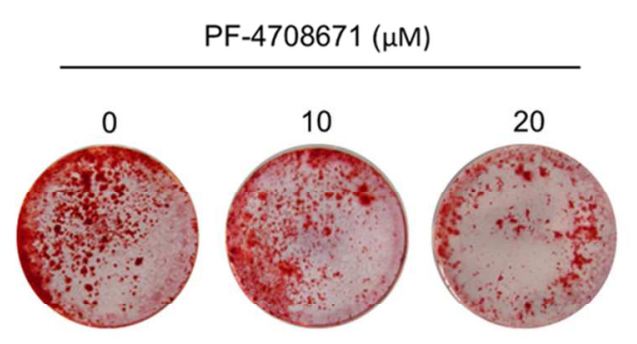

B

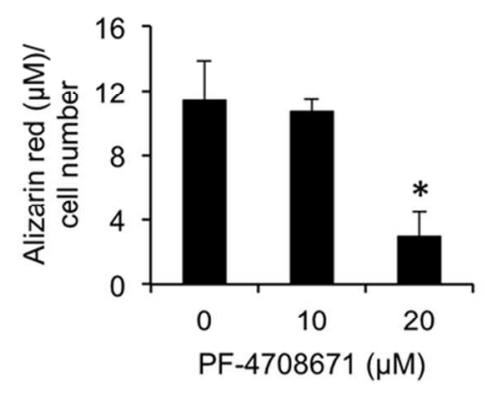

Supplementary FIG. S2. Effect of S6K1 inhibitor PF-4708671 on ATRA-induced osteogenic induction of mASCs. mASCs were induced to undergo osteogenic induction in the absence or presence of PF-4708671 at 10 or $20 \mu \mathrm{M}$ for 15 days. (A) Alizarin red staining of PF-4708671-treated mASCs 15 days post osteogenic induction. (B) Extracted Alizarin red stain was quantified and normalized to cell number. $* p<0.01$ as compared to untreated mASCs using one-way ANOVA. $43 \times 18 \mathrm{~mm}(600 \times 600 \mathrm{DPI})$ 